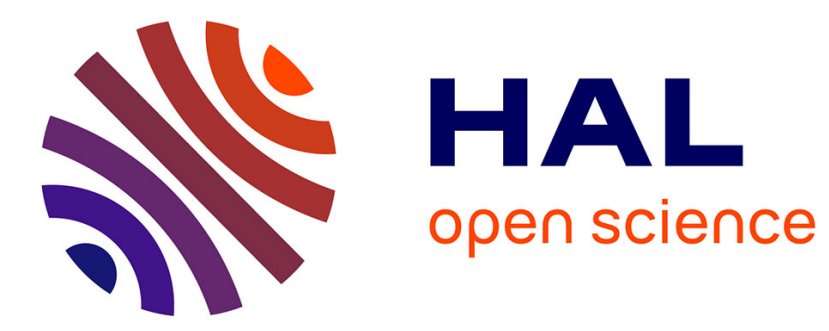

\title{
The value of biodiversity as an insurance device
}

\author{
Emmanuelle Augeraud-Véron, Fabbri Giorgio, Katheline Schubert
}

\section{To cite this version:}

Emmanuelle Augeraud-Véron, Fabbri Giorgio, Katheline Schubert. The value of biodiversity as an insurance device. 2018. hal-01779333

\section{HAL Id: hal-01779333 \\ https://hal.science/hal-01779333}

Preprint submitted on 26 Apr 2018

HAL is a multi-disciplinary open access archive for the deposit and dissemination of scientific research documents, whether they are published or not. The documents may come from teaching and research institutions in France or abroad, or from public or private research centers.
L'archive ouverte pluridisciplinaire HAL, est destinée au dépôt et à la diffusion de documents scientifiques de niveau recherche, publiés ou non, émanant des établissements d'enseignement et de recherche français ou étrangers, des laboratoires publics ou privés. 


\section{GAEL \\ Grenoble Applied Economic Laboratory}

Consumption - Energy - Innovation

The value of biodiversity as an insurance device

Augeraud-Véron, E.

Fabbri, G.

Schubert, $\mathrm{K}$.

April, 17, 2018

JEL: Q56, Q58, Q10, Q15, Q13, O20, C73 


\title{
The value of biodiversity as an insurance device*
}

\author{
E. Augeraud-Véron $†$ G. Fabbri $;$ K. Schubert ${ }^{\S}$
}

April 17, 2018

\begin{abstract}
This paper presents a benchmark stochastic endogenous growth model of an agricultural economy. Producing food requires land, and increasing the share of total land devoted to farming mechanically reduces the share of land devoted to biodiversity conservation. However, safeguarding a greater number of species guarantees, through spatial exchanges, better ecosystem services, which in turn ensure lower volatility of agricultural productivity. The optimal conversion/conservation rule is explicitly characterized, as well as the total value of biodiversity in terms of the welfare gain from biodiversity conservation, and the marginal value of biodiversity in terms of risk premium reduction, namely its insurance value. The Epstein-Zin-Weil specification of preferences allows us to disentangle the effects of risk aversion and aversion to fluctuations.
\end{abstract}

KEY WORDS: Biodiversity, stochastic endogenous growth, insurance value, recursive preferences.

JEL CLASSIFICATION: Q56, Q58, Q10, Q15, O13, O20, C73.

*This work was partially supported by the project L'économie de la biodiversité dans un modèle de culture optimal au sein du PEPS Humanité - Mathématiques - sciences de l'Information - CNRS - France. The research of Giorgio Fabbri has been developed in the framework of the center of excellence LABEX MMEDII (ANR-11-LABX-0023-01). The authors thank Aude Pommeret and François Salanie for their kind and insightful comments on an early version of this paper. All remaining errors are of course theirs.

${ }^{\dagger}$ GREThA, Université de Bordeaux, France emmanuelle.augeraud@u-bordeaux.fr.

${ }^{\ddagger}$ Univ. Grenoble Alpes, CNRS, INRA, Grenoble INP, GAEL, 38000 Grenoble, France. giorgio.fabbri@univgrenoble-alpes.fr.

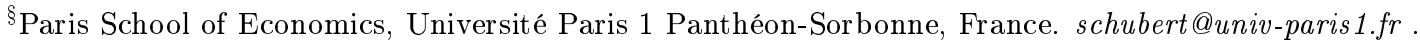




\section{Introduction}

From 1999 to 2008, 48000 square kilometers of wildland were turned into cropland. By 2013, cropland covered $12 \%$ of earth's ice-free surface; annually, more than $10 \%$ of the world net primary production is turned into crops (Phalan et al. [31]). As documented since a long time by ecologists (Preston [32], Rosenzweig [36]), the ensuing destruction of natural habitats causes species extinctions and thus biodiversity loss. This destruction of habitats and natural ecosystems for agricultural purposes, useful and inevitable as it may appear, is increasingly questioned. Indeed, biodiversity provides for free a wide range of goods and services: provisioning services, such as food, wood, freshwater, and regulating services, such as control of the local climate, clean water provision, flood control, regulation of soil fertility, pollination, or biocontrol (pest control, resistance to plant invasion, disease control). These services are impossible or too costly to replace artificially. They have a multifaceted non-market value, which key determinants are important to understand for the design of conservation policies. This paper offers a contribution to the theoretical economics literature on biodiversity, which is at the moment rather scarce, in contrast to the rapidly growing empirical literature. It does not pretend to explain the overall value of biodiversity, but focuses the attention to one of the possible components of this overall value, the insurance component, in the context of land use change.

In the debate about the trade-off between food production and biodiversity conservation, a growing body of evidence shows that, ironically, one of the ecological and economic determinants of the value of ecosystem services is the feedback effect that biodiversity destruction exerts on agricultural productivity. Biodiversity destruction negatively impacts the climatic, hydrological and, more generally, ecological environment, which may in turn negatively affect the mean level and/or the variability of agricultural productivity over time (Fuglie and Nin-Pratt [13], de Mazancourt et al. [6]). To put things differently, biodiversity conservation can provide beneficial services that enhance mean agricultural productivity and reduce its variability. We are interested in the second of these effects, namely the ability of a high level of biodiversity to dampen fluctuations in agricultural productivity around its trend. In this context, biodiversity acts as a form of insurance, a natural insurance against fluctuations in agricultural production. 
To better study the impact of risk on optimal decisions in a dynamic context, we follow the approach pioneered by Epstein and Zin (Epstein and Zin [9], [10], Duffie and Epstein [7]) and represent preferences by a recursive utility function. In this way, we are able to disentangle two logically distinct concepts, the attitude towards time, represented by the aversion to intertemporal fluctuations parameter, and the attitude towards risk, represented by the relative risk aversion parameter. Risk aversion quantifies the preference for certain rather than uncertain outcomes, and it only makes sense in a stochastic context. Conversely, aversion to intertemporal fluctuations, i.e. the inverse of the elasticity of intertemporal substitution, measures the propensity to smooth consumption over time, and is a fundamental parameter in deterministic dynamic models as well. In typical endogenous growth models, when the growth rate is positive, aversion to intertemporal fluctuations takes the form of a willingness to increase the level of consumption at the expense of its growth rate. Several studies ${ }^{1}$ in the field of natural resources and the environment show that these two concepts cannot satisfyingly be embodied in a single parameter as they are when intertemporal additive expected utility is considered.

We characterize the optimal allocation of land to biodiversity conservation. We show that the optimal share of land devoted to biodiversity conservation is constant over time. It decreases with the social discount rate and increases with risk aversion. It decreases with aversion to fluctuations when the average trend of agricultural productivity corrected by the elasticity of total volatility to the share of land devoted to farming is larger than the discount

\footnotetext{
${ }^{1}$ In a model of reservoir management, Howitt et al. [18] show that the intertemporal additive expected utility function does not fit their data, whereas the recursive utility function does. Peltola and Knapp [30] use recursive utility to study forestry management, and Lybbert and McPeak [28] for the trade-off among different livestock for Kenyan pastoralists. They empirically identify the distinct values that should be taken by the intertemporal elasticity of substitution and risk aversion parameters. Ha-Duong and Treich [17] evaluate policies in a context of global warming; it is shown that the optimal policy responds differently to variation in the intertemporal substitution parameter and in the risk aversion one. The same result is observed by Knapp and Olson [20] for rangeland and groundwater management. They consider the effect of both parameters on the optimal decision rule, showing in particular that when intertemporal substitution has a major effect, risk aversion does not impact the optimal policy. The different roles of the two parameters is also proved in Epaulard and Pommeret [8] in the context of extraction of a non-renewable resource in a continuous time framework.
} 
rate, but increases with aversion to fluctuations in the opposite case.

We then compute the total and the marginal values of biodiversity, interpreted as an insurance against the volatility of agricultural productivity, because hedging against these fluctuations is the only role biodiversity has in our model. The total value of biodiversity is defined with reference to Lucas [26], [27] as the welfare gain from biodiversity conservation under the optimal solution compared to a solution where total land conversion is achieved ans all biodiversity is lost. Probably more meaningful in our context is the marginal welfare gain associated to a biodiversity increase. It has been analyzed in a series of groundbreaking studies by Baumgärtner [3], Quaas et al. [33], Quaas and Baumgärtner [34], Baumgärtner and Quaas [4] and Baumgärtner and Strunz [5]. We therefore compute the marginal value of biodiversity, in the spirit of Baumgärtner [3]. We study the determinants of these two values of biodiversity. We show that both are increasing functions of risk aversion, but that the effect of aversion to fluctuations is ambiguous. When the average trend of agricultural productivity corrected by the elasticity of total volatility to the share of land devoted to farming is smaller than the discount rate, the signs of the effects of increasing aversion to fluctuations on the share of land devoted to biodiversity conservation and on the total and marginal values of biodiversity are, quite intuitively, the same. But a counter-intuitive result may appear in the opposite case, when risk parameters are large: the two signs may be opposite. Then, when society becomes more averse to fluctuations it preserves less and less of something that has more and more value: biodiversity.

Our analysis contributes to two strands of the literature. First, we contribute to a nascent theoretical literature on the insurance value of biodiversity in the sense of Baumgärtner [3], enlarging its definition to a stochastic dynamic framework. Indeed, the static context is a limitation, because the effects of biodiversity degradation accumulate and spread over time. In our model, the volatility of agricultural productivity depends on the whole historical path of biodiversity conservation decisions. This dynamic context is essential to being able to speak about elasticity of intertemporal substitution and aversion to fluctuations.

The second strand that our paper contributes to is the small but growing literature on natural resources that disentangles intertemporal substitution and risk aversion. In the specific case of the relation between optimal growth and biodiversity conservation, we show that the 
optimal allocation of land and the values of biodiversity respond qualitatively differently to the two parameters, and highlight the sometimes discordant influences of preferences towards risk and time.

The paper proceeds as follows. In section 2 we present the basics of the model. We explicitly solve it to obtain the optimal land conversion rate in section 3 , and we compute the total and marginal values of biodiversity in section 4. Section 5 concludes. All the proofs of the results are in the Appendix.

\section{The model}

We build a highly stylized general equilibrium model of an agricultural economy, the simplest we can think of that allows us to compute the value of biodiversity as insurance against agricultural productivity fluctuations. We first present the model and its optimal solution, and then determine the total and marginal values of biodiversity.

The planner of the agricultural economy, or the unique producer-consumer living in this economy (the farmer), owns a stock $L=1$ of land and she has to decide how to allocate it between two possible intended uses: farming and biodiversity conservation. For $t \geq 0$, we denote by $f(t) \in[0,1]$ and $1-f(t) \in[0,1]$ the shares of land respectively used for farming and left undeveloped, that is devoted to biodiversity conservation.

In the modeling of the ecological part of the model we have to specify the relationship between space and biodiversity. Eppink and Withagen [11] simply introduce the classical species-area curve first proposed in the 1920s by O. Arrhenius [2] and H. Gleason [14] to describe this relationship. In Smulders et al. [38], biodiversity depends on the carrying capacity, which is itself a function of the size of habitat. In $\mathrm{Li}$ et al. [24], the number of species is explicitly modelled. We choose here to follow Lafuite and Loreau [23].

First, the evolution of biodiversity $B(t)$, defined as the variety of ecological elements present in a place, including genes, species, functional traits, communities etc., can be modeled $\operatorname{as}^{2}$ :

$$
\eta \dot{B}(t)=-(B(t)-S(t)) \quad \text { with } \quad \eta>0,
$$

\footnotetext{
${ }^{2}$ See Lafuite and Loreau [23] and the references therein.
} 
where $S(t)$ is the long term species richness. This dynamics is very slow, compared to the time scale of agricultural activity, so that we can safely consider that the dynamics occurs on the slow manifold $B(t)=S(t)$.

Second, according to the species-area curve, the number of species $S(t)$ is constrained by the size of the natural habitat:

$$
S(t)=\epsilon_{1}(1-f(t))^{\epsilon_{2}} \quad \text { with } \quad \epsilon_{1}>0 \quad \text { and } \quad 0<\epsilon_{2}<1 \text {. }
$$

The economic part of the model is very simple. We assume that agricultural production at time $t$ is given by:

$$
Y(t)=f(t) A(t)
$$

where $A(t)$ is the productivity of a unit of land devoted to farming at time $t$, which dynamics is described by a stochastic differential equation (SDE). More precisely, given a complete probability space $(\Omega, \mathcal{F}, \mathbb{P})$ and a real standard Brownian motion $W:[0,+\infty) \times \Omega \rightarrow \mathbb{R}$, adapted to some filtration $\mathcal{F}_{t}$, we assume that $A(t)$ is a solution of the following SDE:

$$
\left\{\begin{array}{l}
\mathrm{d} A(t)=\alpha A(t) \mathrm{d} t+\Sigma(t) A(t) \mathrm{d} W(t) \\
A(0)=A_{0} .
\end{array}\right.
$$

In such an expression, $\alpha \in \mathbb{R}$ represents some fixed and exogenous rate of technological progress in farming activities (it can be equal to 0 ), and the term $\Sigma(t)$ measures the volatility of agricultural productivity.

Finally, we suppose that at each time $t \geq 0$ all the production is consumed, so that:

$$
C(t)=Y(t)
$$

This assumption is not innocuous. It implies that there is no precautionary saving: the farmer cannot store or save part of its agricultural production to hedge against the risk of poor future productivity.

The link between the ecological and the economic parts of the model is given by the specification of the volatility of agricultural productivity $\Sigma(t)$. This volatility has two components. The exogenous component $\sigma>0$ represents the intrinsic volatility, stemming for instance from weather events (floods, droughts, etc.). The endogenous component is a decreasing function of the level of biodiversity $B(t)$. The justification of this assumption can be found 
in Loreau et al. [25], who show that "biodiversity provides spatial insurance for ecosystem functioning by virtue of spatial exchanges among local systems in heterogeneous landscapes." Two mechanisms are brought out: spatial averaging and functional compensation. In our framework, those exchanges take place between a natural biodiversity-rich ecosystem, namely the part of land left undeveloped, and the simplified agricultural ecosystem to be found in the part of land converted to farming. The spatial exchanges dampen the fluctuations of the productivity of the agricultural activity. Overall, to represent those effects, and using the link between biodiversity and the share of land devoted to farming highlighted in the ecological part of the model (equations (1) and (2)), we specify total volatility as:

$$
\Sigma(t)=\sigma g(B(t))=\sigma f(t)^{\chi / 2} \quad \text { with } \quad \chi>0 .
$$

Total volatility decreases as the land devoted to biodiversity conservation, and then biodiversity itself, increases. It is in this sense that biodiversity appears in the model as insurance against adverse outcomes. We finally get that the productivity dynamics is described by the following SDE:

$$
\left\{\begin{array}{l}
\mathrm{d} A(t)=\alpha A(t) \mathrm{d} t+\sigma f^{\frac{\chi}{2}} A(t) \mathrm{d} W(t) \\
A(0)=A_{0} .
\end{array}\right.
$$

It should be noted that, because of the different time scales of agricultural activity and biodiversity evolution ${ }^{3}$, we do not include in the state equation any ecosystem transitional internal dynamics. We also disregard possible conversion costs and irreversibility of biodiversity depletion. Of course the need to keep the model analytically tractable is not unconnected with this choice but we can observe that ex post this choice is not too "heroic". Indeed along the optimal path the decision maker always chooses to keep the same shares of the land for farming and for biodiversity conservation (see Propositions 3.2 and 3.5). This suggests that adding conversion costs or transitional costs/dynamics or an irreversibility constraint on the evolution of biodiversity should not essentially change the results. The situation would be very different if we had found that at the optimum the amount of land devoted to biodiversity conservation changes over time.

The planner maximizes, over the set of the $[0,1]$-valued $\mathcal{F}_{t}$-adapted processes, an aggregate

\footnotetext{
${ }^{3}$ See the comment following equation (1).
} 
social welfare criterion in the form of an infinite horizon, continuous time, Epstein-Zin-Weil utility function characterized by a constant relative risk aversion of $\theta, \theta>0, \theta \neq 1$, an intertemporal elasticity of substitution of $\phi^{-1}>0$ and a discount rate of $\rho>0$. Recall that the case $\theta=\phi$ corresponds to the usual time additive expected utility function. The inverse of the intertemporal elasticity of substitution $\phi$ can also be interpreted as a measure of aversion to fluctuations, as an agent with a high $\phi$ prefers to smooth consumption over time ${ }^{4}$. Of course, $\phi$ and $\theta$ are distinguished because they represent logically different concepts: the attitude towards time and the attitude towards risk, respectively.

By definition of the Epstein-Zin utility function in continuous time, utility at time $t, u(t)$, depends on current consumption $C(t)$ and a certainty equivalent of the distribution of time $t+d t$ utility, conditioned upon time $t$ information:

$$
u(t)=f\left(C(t), \mu_{t}(u(t+d t))\right)
$$

where $f$ is the aggregator and $\mu_{t}(u(t+d t))$ the certainty equivalent.

For instance we can choose as in Epstein and Zin [10], Svensson [39] and Duffie and Epstein [7] a CIES aggregator:

$$
f(C, v)=\left(C^{1-\phi}+e^{-\rho d t} v^{1-\phi}\right)^{\frac{1}{1-\phi}}, \quad \phi \neq 1 .
$$

When the attitude towards risk is modeled by the CRRA utility function $\frac{C^{1-\theta}}{1-\theta}, \theta>0$ and $\theta \neq 1$, the certainty equivalent is defined by:

$$
\mu_{t}(x)=\left[\mathbb{E}_{t} x^{1-\theta}\right]^{\frac{1}{1-\theta}}, \quad \theta \neq 1
$$

It yields:

$$
u(t)=\left(C(t)^{1-\phi} d t+e^{-\rho d t}\left[\mathbb{E}_{t} u(t+d t)^{1-\theta}\right]^{\frac{1-\phi}{1-\theta}}\right)^{\frac{1}{1-\phi}} .
$$

As it has been shown in Duffie and Epstein [7] that the utility function $U(t)$ defined by $U(t)=\frac{u(t)^{1-\theta}}{1-\theta}$ yields the same optimal path as the utility function $u(t)$, the previous equation can be replaced by ${ }^{5}$ :

$$
(1-\theta) U(t)=\left(C(t)^{1-\phi} d t+e^{-\rho d t}\left[(1-\theta) \mathbb{E}_{t} U(t+d t)\right]^{\frac{1-\phi}{1-\theta}}\right)^{\frac{1-\theta}{1-\phi}} .
$$

\footnotetext{
${ }^{4}$ If $\theta>\phi$, individuals are more risk-averse than concerned about consumption smoothing. In this case (Gollier [15]), an agent is said to have Preferences for an Early Resolution of Uncertainty (PERU), notably the case among poor, vulnerable populations (Lybbert and MacPeak [28]).

${ }^{5}$ See also Smith [37].
} 
We denote by $V\left(A_{0}\right)$ the value function of the described problem.

\section{The optimal land conversion rate}

The following conditions, which we will always suppose to be verified in the sequel of the paper, will be shown to be necessary to explicitly express the value function and ensure that it remains finite.

Hypothesis 3.1. The parameters satisfy the following conditions:

$$
\text { (i) } \quad \chi \geq 1, \quad \text { (ii) } \quad \rho>\alpha(1-\phi), \quad \text { (iii) } \quad \rho\left(1+\frac{\phi-\theta}{\chi}\right)>\alpha(1-\theta) .
$$

Condition (i) in (9) postulates that the instantaneous total variance $\frac{\Sigma^{2}}{2}=\frac{\sigma^{2} f^{\chi}}{2}$ is (weakly) convex in $f$. When this condition is verified, the marginal effectiveness of an increase of the share $1-f$ of the land devoted to biodiversity conservation in terms of volatility reduction is decreasing. We will show in particular (see the proof of Proposition 3.2) that this condition, together with inequality (ii) in (9), form the feasibility condition, insuring that the optimal control is positive. Condition (ii) imposes that the discount rate is high enough. Notice that the parameter that matters for the feasibility condition is the aversion to fluctuation $\phi$ and not the risk aversion $\theta$. Also, condition (ii) is always satisfied when $\phi>1$. Observe that feasibility conditions are not required in the separable preference specification of the model (when $\phi=\theta$ ) because in this case the infinite derivative of the instantaneous utility at zero is enough to ensure the decision maker always chooses a strictly positive consumption and then a strictly positive $f$. The situation changes when the intertemporal structure is more complex so a condition to ensure the existence of an optimal admissible strategy is necessary. We can, formally, verify this fact observing that (ii) reduces, when $\phi=\theta$, to condition (iii), the transversality condition of the problem. This is a rather general fact which also arises in standard optimal investment models (see Smith [37]).

The transversality condition (iii) in (9) gives the restrictions on the parameters ensuring that the value function remains finite at infinity. Its economic intuition is not very transparent at a first look but it does not need to be so. Indeed, as soon as we depart from the the standard separable preference case $(\phi=\theta)$ several possible ordinally equivalent representations of 
Epstein-Zin preferences are possible (see Duffie and Epstein [7], Section 3.3). All of them (since they are ordinally equivalent) entail the same optimal control but they need different transversality conditions so that, for instance, once we specified the parameters of the economy $\alpha, \chi$ and $\sigma$ and risk and fluctuation aversion parameters $\theta$ and $\phi$, the set of possible discounting $\rho$ which satisfy the transversality condition depends on the chosen representations of EpsteinZin preferences. So the transversality condition is not intrinsic here. Observe that, if we look at the standard separable preference case $(\phi=\theta)$ the condition reduce to $\rho>\alpha(1-\theta)$. It is particularly simple: it is indeed a simplified version of the conditions appearing for instance in standard optimal investment models (see e.g. Merton [29]), and it has the form of the transversality condition for the $A K$ deterministic baseline model (see Acemoglu [1]). The reason of this fact is that, differently from standard stochastic growth models, in our case the choices of the agent do not alter the drift term in the equation describing the dynamics of $A$.

Under Hypothesis 3.1 the value of the positive constant

$$
\frac{\rho-\alpha(1-\phi)}{\frac{\sigma^{2}}{2} \theta(\chi-1+\phi)}
$$

will be important to distinguish between interior and corner solutions. In the two following propositions we will see what happens when this constant is greater or smaller than 1 . We begin by describing the dynamics of the system in the interior solution case.

Proposition 3.2. Let Hypothesis 3.1 be satisfied. Assume that:

$$
\frac{\rho-\alpha(1-\phi)}{\frac{\sigma^{2}}{2} \theta(\chi-1+\phi)}<1
$$

Then the value function of the problem can be written explicitly. It is equal to:

$$
V(A)=\frac{1}{1-\theta} \beta A^{1-\theta}
$$

where

$$
\beta=\left[\left(\frac{\chi-1+\phi}{\rho-\alpha(1-\phi)}\right)^{\frac{\chi-1+\phi}{\chi}} \frac{1}{\chi\left(\theta \frac{\sigma^{2}}{2}\right)^{\frac{1-\phi}{\chi}}}\right]^{\frac{1-\theta}{1-\phi}} .
$$

The optimal control is constant and deterministic, and it is given by:

$$
f^{*}(t)=f^{*}:=\left(\frac{\rho-\alpha(1-\phi)}{\frac{\sigma^{2}}{2} \theta(\chi-1+\phi)}\right)^{\frac{1}{\chi}}, \quad \text { for any } t \geq 0 .
$$

Finally, (9) guarantees the respect of the feasibility and transversality conditions. 
Proof. See Appendix. ${ }^{6}$

Observe that (9) and (10) imply

$$
\alpha(1-\phi)<\rho<\alpha(1-\phi)+\theta \frac{\sigma^{2}}{2}(\chi-1+\phi)
$$

For given $\alpha$ and $\phi$, the range of the admissible discount rates for the existence of an optimal interior solution is all the larger since the parameters characterizing risk in this economy, namely risk aversion $\theta$, the intrinsic volatility $\sigma$, and the elasticity of total volatility with respect to the share of land devoted to farming, $\chi$, are high.

We now detail how the different parameters of the model, and in particular the preference and technological parameters, affect the total value of biodiversity.

Proposition 3.3. The optimal conversion rate $f^{*}$ is an increasing function of the discount rate $\rho$, a decreasing function of the intrinsic volatility of agricultural productivity $\sigma$, and a decreasing function of risk aversion $\theta$. It is also a decreasing function of aversion to fluctuations $\phi$ if $\alpha \chi-\rho<0$, but an increasing function of aversion to fluctuations if $\alpha \chi-\rho>0$.

Proof. Straightforward derivations of (13) give the results.

The first three results are consistent with intuition. The higher the discount rate, that is, the more impatient a society is, the less it cares about the future and the less it wants to insure against future uncertainty. Such a society has a strong incentive to convert a large part of its land to agriculture to enjoy food consumption now. Conversely, the higher the

\footnotetext{
${ }^{6}$ The arguments of the proof of Proposition 3.2 can be adapted to a more general case where, instead of considering $\Sigma$ as in (6), we consider the situation where $\Sigma(t)=\sigma \gamma(f(t))$ for some continuous function $\gamma(\cdot)$. In this case one can show that, if the couple $\left(\beta_{\gamma}, f_{\gamma}^{*}\right) \in(0,+\infty) \times[0,1]$ is a solution of the following system:

$$
\left\{\begin{array}{c}
f_{\gamma}^{*} \in \arg \max _{f \in[0,1]}\left(\frac{f^{1-\phi}}{\beta_{\gamma}^{\frac{1-\phi}{1-\theta}}}-(1-\phi) \gamma(f)^{2} \frac{\sigma^{2}}{2} \theta\right) \\
\rho-\alpha(1-\phi)=\left(\frac{f_{\gamma}^{* 1-\phi}}{\beta_{\gamma}^{\frac{1-\phi}{1-\theta}}}-(1-\phi) \gamma\left(f_{\gamma}^{*}\right)^{2} \frac{\sigma^{2}}{2} \theta\right)
\end{array}\right.
$$

and if some suitable feasibility and transversality conditions are verified, the optimal land used for farming is $f_{\gamma}^{*}$ and the optimal social welfare is $\frac{1}{1-\theta} \beta_{\gamma} A^{1-\theta}$. The problem is that this result is not at all explicit: we cannot express $f_{\gamma}^{*}$ and the value of biodiversity in terms of the various parameters of the model and qualitatively describe their behavior. For this reason we decided to concentrate our analysis on the case $\gamma(f)=f^{\chi / 2}$.
} 
intrinsic volatility and the more risk-averse a society is, the more it wants to insure against future uncertainty.

Things are more complex regarding the effect of society's aversion to fluctuations. When the difference between the trend of agricultural productivity corrected by the elasticity $\chi$ and the discount rate is negative, future prospects are - on average - rather poor, and at the same time the society is impatient. Both effects lead to better present than future outcomes. A society averse to fluctuations logically wants to counteract these forces, and is thus willing to insure against adverse outcomes in the future by conserving more biodiversity. The opposite occurs when $\alpha \chi-\rho>0$.

Notice than when $\alpha \chi>\rho$, increasing both risk aversion and aversion to fluctuations has an ambiguous effect on the optimal conversion rate, since the two parameters characterizing preferences act in opposite directions.

In the situation described in Proposition 3.2 a complete description of the optimal dynamics of the system can be provided. What we have is the following corollary of the previous result.

Corollary 3.4. Let the assumptions of Proposition 3.2 be satisfied. Then the optimal evolution of $A(t)$ and $C(t)$ are respectively:

$$
A(t)=A_{0} \exp \left[\left(\alpha-\frac{\sigma^{2}}{2} f^{* \chi}\right) t+\sigma f^{* \frac{\chi}{2}} W(t)\right]
$$

and:

$$
C(t)=f^{*} A(t)
$$

In particular

$$
\mathbb{E}[A(t)]=A_{0} e^{\alpha t}, \quad \operatorname{Var}[A(t)]=A_{0}^{2} e^{2 \alpha t}\left(e^{\sigma^{2} f^{* \chi} t}-1\right)
$$

The dynamics of the optimal land productivity described in (14) is a geometric Brownian motion, so that at any time $t$ the distribution of $A(t)$ is log-normal and has, respectively, the expected value and the variance described in (15). Given the expression of the dynamics of $A$ in (7), the growth rate of the expected value of $A(t)$ only depends on the parameter $\alpha$, while $f^{*}$ positively impacts its variance. 
We can now return to the results of Proposition 3.3 and look more closely at their significance. First, the effect of increased risk aversion can be seen as a form of precautionary saving effect. Indeed, increasing $\theta$ has the consequence of reducing $f^{*}$ which, on the one hand, decreases the (certain) consumption level today but, on the other hand, increases the average value of the consumption growth rate $^{7}$ that is given by $\mathbb{E}[g]=\alpha-\frac{\sigma^{2}}{2} f^{* \chi}$.

Second, increasing society's aversion to fluctuations also generates a level effect and a growth effect. The situation is similar to that of the standard deterministic benchmark $A K$ growth model. The effect of $\phi$ depends on the value of $\alpha \chi-\rho$. When it is positive, the bigger $\phi$ is, the higher the initial consumption $C(0)=f^{*} A(0)$ is, and the lower the average value of the growth rate $\mathbb{E}[g]=\alpha-\frac{\sigma^{2}}{2} f^{* \chi}$. The opposite happens when $\alpha \chi-\rho<0$.

We now characterize the value function in the corner solution case.

Proposition 3.5. Let conditions (i) and (ii) of (9) be satisfied. Assume in addition that

$$
\frac{\rho-\alpha(1-\phi)}{\frac{\sigma^{2}}{2} \theta(\chi-1+\phi)} \geq 1
$$

and

$$
\rho>(1-\theta)\left(\alpha-\theta \frac{\sigma^{2}}{2}\right) .
$$

Then the value function of the problem can be written explicitly. It is equal to:

$$
V_{C}(A)=\frac{1}{1-\theta} \beta_{C} A^{1-\theta}
$$

where

$$
\beta_{C}:=\left[\rho-(1-\phi)\left(\alpha-\theta \frac{\sigma^{2}}{2}\right)\right]^{-\frac{1-\theta}{1-\phi}} .
$$

Moreover the optimal control is constant and deterministic, and it is given by:

$$
f^{*}(t)=f^{*}:=1, \quad \text { for any } t \geq 0
$$

Proof. See Appendix.

\footnotetext{
${ }^{7}$ According to equation (14), the average value of the growth rate is given by $\frac{1}{t} \mathbb{E}\left[\left(\alpha-\frac{\sigma^{2}}{2} f^{* \chi}\right) t+\sigma f^{* \frac{\chi}{2}} W(t)\right]=\alpha-\frac{\sigma^{2}}{2} f^{* \chi}$. It should not be confused with the value of the growth rate of the average value of $C(t)$, which is $\alpha$, as shown in (15).
} 
As underlined by the previous results, the structure of the value function is the same in the two cases (i.e. both are homogeneous of degree $1-\theta$ ), but of course the multiplicative constants differ. A corollary similar to Corollary 3.4 can be obtained in the corner case: the optimal dynamics of $A(t)$ is described by (14) where, instead of $f^{*}$, we have 1 .

Condition (17) is the transversality condition of the problem. Notice that (16) and (17) imply $\rho>(1-\phi)\left(\alpha-\theta \sigma^{2} / 2\right)$, meaning that $\beta_{C}$ in (19) is well defined.

\section{The value of biodiversity}

In our model, the value of biodiversity comes exclusively from its ability to dampen the fluctuations of agricultural productivity. A wide range of ecosystem services provided for free by biodiversity, whether they are provisioning or regulating services, are ignored, as well as the amenity value of biodiversity. Therefore it is clear that our aim here is not to evaluate the overall value of biodiversity. It is much more modest. We want to make precise what the value of biodiversity is, in its function of hedging against the volatility of agricultural productivity. To do so, we follow two different directions. First, we build on the famous papers by Lucas [26], [27] on the welfare cost of fluctuations to compute the total value of biodiversity defined as the welfare gain from biodiversity conservation. Second, we compute the insurance value of biodiversity, defined as the marginal value of biodiversity in its risk premium reduction function, in the spirit of Baumgärtner [3]. The first value is total while the second one is marginal and, as such, is probably more relevant. Indeed, the meaningful decision to be made in the real world at a given moment is not whether to conserve the optimal level of biodiversity or to destroy all biodiversity, but rather whether to decrease marginally biodiversity to increase the land devoted to farming.

\subsection{The welfare gain from biodiversity conservation}

Following Lucas [26], [27], we define and compute the total value of biodiversity as the welfare gain from biodiversity conservation. According to Lucas, the welfare cost of fluctuations is the percentage increase in consumption needed at all dates to compensate the representative agent for the presence of fluctuations, i.e. to make him indifferent between the actual consumption 
path, subject to fluctuations, and the corresponding deterministic consumption path. In the same spirit, we define here the welfare gain from biodiversity conservation as the percentage increase in consumption that a society considers an acceptable compensation for exchanging the optimal situation for a situation with nil biodiversity and all land used for farming. It is thus defined as follows:

Definition 4.1. The welfare gain from biodiversity conservation (i.e. the total value of biodiversity) is the percentage increase in consumption society is willing to accept at all dates to give up the optimal level of biodiversity in favor of no biodiversity at all.

The value function in the no-biodiversity case is denoted by $V_{B}(A)$ and is characterized in the following proposition. Let $\lambda$ be the welfare gain defined above. According to Definition 4.1, $\lambda$ satisfies:

$$
V(A)=V_{B}((1+\lambda) A)
$$

Observe that when (16) is satisfied, i.e. when we are at the optimum in the corner case $f^{*}=1$, the optimal and the no-biodiversity solution are equivalent, so in this section we assume that (10) is verified, i.e. that we are in the interior case at the optimum. We will also make a technical assumption to be able to characterize the explicit form of the welfare in the no-biodiversity case.

Proposition 4.2. Let conditions (i) and (ii) of (9) and condition (10) be satisfied and suppose that:

$$
\rho>(1-\phi)\left(\alpha-\theta \frac{\sigma^{2}}{2}\right)
$$

and

$$
\rho>(1-\theta)\left(\alpha-\theta \frac{\sigma^{2}}{2}\right)
$$

Then the welfare in the no-biodiversity case is given by:

$$
V_{B}(A)=\frac{1}{1-\theta} \beta_{B} A^{1-\theta}
$$

where

$$
\beta_{B}:=\left[\rho-(1-\phi)\left(\alpha-\theta \frac{\sigma^{2}}{2}\right)\right]^{-\frac{1-\theta}{1-\phi}}
$$

Proof. See Appendix. 
Conditions (21) and (22) are the counterparts, respectively, of the feasibility and of the transversality conditions in Proposition 3.2 (we diffusely described their meaning in the paragraphs following (9)). They reduce to the same condition when $\theta=\phi$ (the standard separable case). Condition (10) together with condition (iii) of (9) imply (22) when $\theta \in(0,1)$ (the reasons is that the utility along the optimal trajectory can be used to bound from above the utility of the no-biodiversity scenario). When $\theta>1$ a specific condition (i.e. (22)) is needed to prevent the utility in the no-biodiversity scenario to be $-\infty$.

Proposition 4.3. Let the assumptions of Proposition 4.2 be satisfied. The welfare gain from biodiversity conservation is:

$$
\lambda=\left(\frac{(\chi-1+\phi) f^{* \chi}+(1-\phi)}{\chi f^{*(\chi-1+\phi)}}\right)^{\frac{1}{1-\phi}}-1 .
$$

Proof. See Appendix.

A comparative statics exercise yields the following results.

Proposition 4.4. Let the assumptions of Proposition 4.2 be satisfied. The total value of biodiversity $\lambda$ is an increasing function of the intrinsic volatility of agricultural productivity and of risk aversion. However, the effect of aversion to fluctuations on the total value of biodiversity is ambiguous.

Proof. See Appendix.

The first two results are intuitive and fit well with the effects of intrinsic volatility and risk aversion on the conversion rate. Higher intrinsic volatility of agricultural productivity and higher risk aversion result in a lower optimal conversion rate, i.e. more biodiversity conservation, and a higher total value of biodiversity, i.e. a higher welfare gain from biodiversity conservation. More is conserved of an asset that is more valued.

To further investigate the role of aversion to fluctuations, it is useful to examine the case where the optimal conversion rate is very high $\left(f^{*}\right.$ close to 1$)$.

Lemma 4.5. Let the assumptions of Proposition 4.2 be satisfied. For $f^{*}$ close to 1 ,

$$
\lambda \simeq \frac{\chi-1+\phi}{2}\left(1-f^{*}\right)^{2}
$$


and

$$
\frac{d \lambda / \lambda}{d \phi / \phi} \simeq \frac{\phi}{\chi-1+\phi}\left[1-2 \frac{f^{*}}{1-f^{*}} \frac{\alpha \chi-\rho}{\chi(\rho-\alpha(1-\phi))}\right]
$$

Proof. See Appendix.

When the optimal conversion rate is close to 1 , the value of biodiversity is proportional to the square of the share of the land optimally devoted to conserving biodiversity.

Besides, if we look at equation (26), we can see that two effects are at work: on the one hand, a direct effect (the term $(\chi-1+\phi) / 2)$ through which increasing $\phi$ increases $\lambda$, and on the other hand, an indirect effect (the term $\left.\left(1-f^{*}\right)^{2}\right)$ of the same sign as the effect of $\phi$ on the share of land devoted to biodiversity conservation. When the corrected trend of productivity $\alpha \chi$ is higher than the discount rate $\rho$, the two effects are discordant.

At first glance, it may seem that the signs of the effect of increasing aversion to fluctuations (or in fact any other parameter) on the value of biodiversity and on the share of land devoted to biodiversity conservation should be the same. This is actually what happens when the discount rate $\rho$ is higher than the corrected trend of productivity $\alpha \chi$ (see equation (27)). Then, increasing aversion to fluctuations increases both the share of land devoted to biodiversity conservation (see Proposition 3.3) and the value of biodiversity. However, when the discount rate is smaller than the corrected trend of productivity, increasing aversion to fluctuations decreases the share of land devoted to biodiversity conservation, but may either decrease or increase the total value of biodiversity, which is less intuitive.

Simulations allow us to check that these results hold when $f^{*}$ is not assumed to be close to 1 . We postpone the discussion of the simulation results until after the determination of the insurance value of biodiversity.

\subsection{The insurance value of biodiversity}

The welfare gain from biodiversity conservation we identified in Subsection 4.1 can be seen as the total value biodiversity has in the framework of our model. Indeed, it is the welfare loss incurred by society when all biodiversity is lost. We would like now to take a step further and try to understand how to measure a marginal gain associated to a variation in the biodiversity 
level. To do so we will follow the idea (and the definition) of insurance value of biodiversity of Baumgärtner [3], generalizing it in our continuous-time set-up.

Fix a certain (in general, non-optimal) level of biodiversity $f$. The corresponding evolution of productivity is then given by the solution $A_{f}(\cdot)$ of $(7)$ for a constant $f(t)=f$. We classically define the risk premium $\Pi(A(t), f)$ associated to the uncertainty arising in our model as the amount of productivity the decision maker is willing to forego at time $t$ in order to get for sure the expected value of the problem. In other terms, if we denote by $\mathcal{W}(C(\cdot))$ the welfare generated by a consumption process $C(\cdot), \Pi(A(t), f)$ is characterized by the relation

$$
\mathcal{W}\left(f \mathbb{E}\left(\left(A_{f}(\cdot)-\Pi\left(A_{f}(\cdot), f\right)\right)\right)=\mathcal{W}\left(f A_{f}(\cdot)\right) .\right.
$$

Following Baumgärtner [3], the insurance value of biodiversity may be defined as follows:

Definition 4.6. The insurance value of biodiversity is the change of the risk premium due to a marginal change in the level of biodiversity, evaluated at the optimal level of biodiversity: $v(A)=-\left.\frac{\partial \Pi(A, f)}{\partial(1-f)}\right|_{f=f^{*}}$.

An explicit expression for this value is given in the following proposition.

Proposition 4.7. Let the assumptions of Proposition 3.2 be satisfied. The insurance value of biodiversity is:

$$
v(A)=-\left.\frac{\partial \Pi(A, f)}{\partial(1-f)}\right|_{f=f^{*}}=A\left(\frac{\chi-1+\phi}{\chi}\right)^{\frac{1}{1-\phi}} \frac{1}{f^{*}} .
$$

Proof. See Appendix.

Proposition 4.8. Let the assumptions of Proposition 3.2 be satisfied. The insurance value of biodiversity $v(A)$ is an increasing function of the intrinsic volatility of agricultural productivity and of risk aversion. It is also an increasing function of aversion to fluctuations when the discount rate is larger than the corrected trend of productivity. However, in the opposite case, the effect of aversion to fluctuations on the insurance value of biodiversity is ambiguous.

Proof. See Appendix.

Figures 1 and 2 show the optimal share of land devoted to farming and the total and marginal values of biodiversity for different configurations of the parameters, satisfying the 

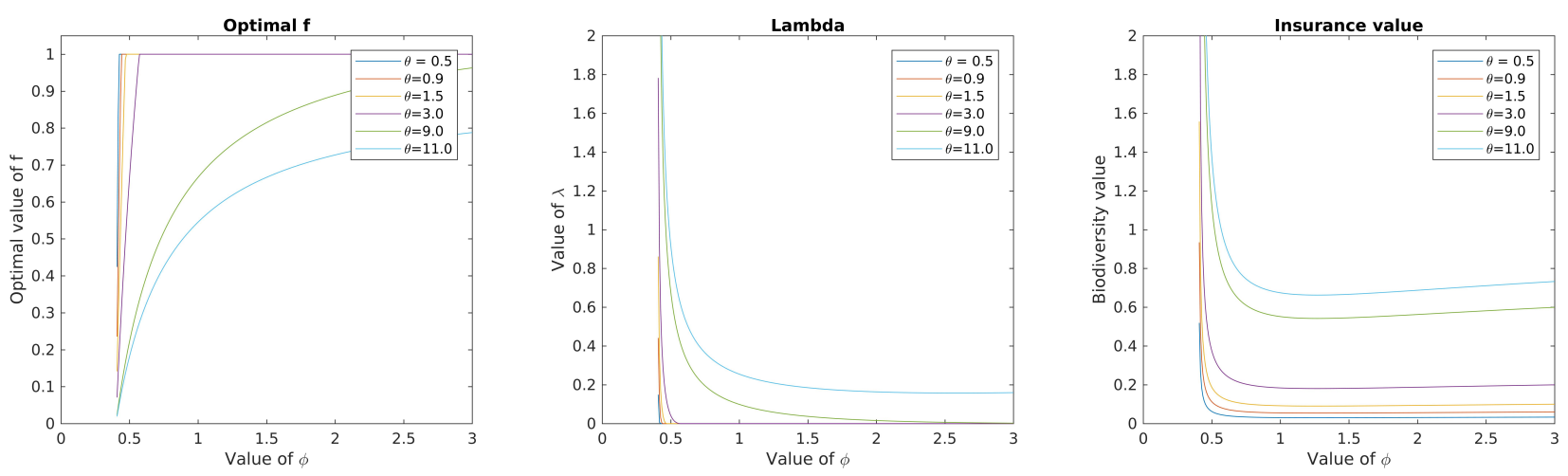

Figure 1: Optimal conversion rate, value of $\lambda$ and insurance value of biodiversity as a function of aversion to fluctuations for $\alpha=0.05, \chi=1$, $\rho=0.03, \sigma=0.1$.

constraints we have determined. We focus on the ambiguous case where $\alpha \chi>\rho$. We have shown that in this case $f^{*}$ is an increasing function of $\phi$, and a decreasing function of $\theta$ and $\sigma$, meaning that the effects of aversion to fluctuations and to risk are discordant. This translates into ambiguous effects on the total and marginal values of biodiversity, $\lambda$ and $v(A) / A$.

On Figures 1 and 2, parameters $\alpha, \chi$ and $\rho$ are fixed. The evolutions of $f^{*}, \lambda$ and $v(A) / A$ when $\phi$ increases are represented for different values of $\theta$ on each figure, combined with a small $\sigma$ on Figure 1 and a larger $\sigma$ on Figure 2. It clearly appears that when the risk parameters $\theta$ and $\sigma$ are large, meaning that the weight of uncertainty and the incentive for insurance are large, $f^{*}$ is small, and $\lambda$ and $v(A) / A$ are U-shaped functions of $\phi$ (Figure 2). The antagonist forces that drive biodiversity conservation then make the optimal amount of land devoted to conservation decrease with the aversion to fluctuations, whereas the total and marginal values of biodiversity increase.

\section{Conclusion}

This paper presents a stylized dynamic model including a particular incentive for biodiversity conservation: its ability to hedge against the volatility of agricultural productivity. Producing food requires land, and increasing the share of total land devoted to farming mechanically 

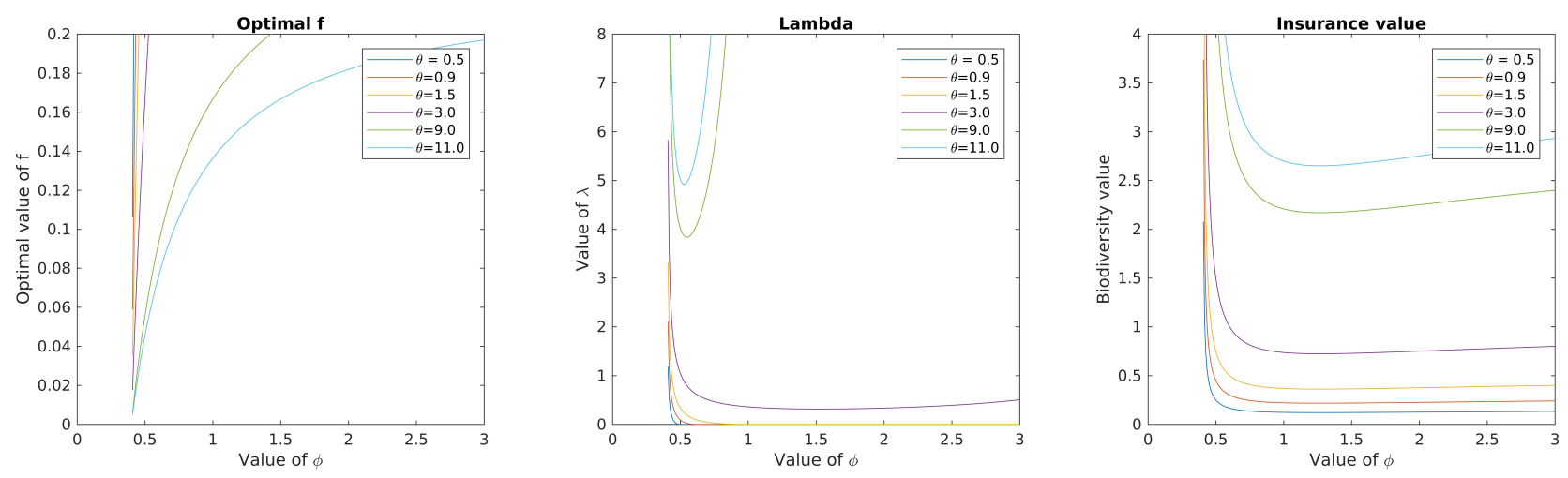

Figure 2: Optimal conversion rate, value of $\lambda$ and insurance value of biodiversity as a function of aversion to fluctuations for $\alpha=0.05, \chi=1$, $\rho=0.03, \sigma=0.2$.

reduces the share of land devoted to biodiversity conservation. However, the safeguarding of a greater number of species ensures through spatial exchanges better ecosystem services pollination, flood control, pest control, etc., which in turn ensure lower volatility of agricultural productivity. The optimal conversion/conservation rule is explicitly characterized, as well as the total value (in terms of the welfare gain from biodiversity conservation) and the marginal value (in terms of risk premium reduction) of biological diversity. The Epstein-Zin-Weil specification of the utility function allows us to disentangle the effects of risk aversion and aversion to fluctuations.

At least two interesting extensions of our work could be explored.

First, there is the debate on land sparing versus land sharing initiated by Green et al. [16]. The question is whether agriculture should be concentrated on intensively farmed land in order to conserve more natural spaces for biodiversity, or whether it should be extensive, less productive, and wildlife-friendly. It has been, and still is, the object of huge debates among ecologists (see for instance Fisher et al. [12], Hulme et al. [19], or Kremen [22]). The dichotomy between the two production schemes depends on the land characteristics and its ability to provide ecosystem services (Renwick and Schellhorn [35]). In its present simple form, the model we use is not suited for adequately studying this question. Indeed, we consider that average productivity growth is exogenous, and in particular independent on 
the level of biodiversity. Biodiversity only affects the volatility of agricultural productivity. These modelling choices allow us to focus our analysis on the insurance value of biodiversity. Besides, we do not introduce artificial inputs, such as fertilizers and pesticides, that could compensate the loss of ecosystem services due to the depletion of biodiversity. A natural extension of the model would be to make average productivity depend on biodiversity and to refine the agricultural production technology. It would allow us to distinguish between the two management practices and their different consequences.

Second, we consider here that the economy does not have access to financial insurance and that there are no means of crop saving/storage. However, if a financial insurance system and/or storage were available, farmers could insure against adverse outcomes by other means than biodiversity conservation. Quaas and Baumgärtner [34] study this problem and show in a static framework that the twos types of insurance (natural and financial) are substitutes. It would be interesting to see whether their result holds in a dynamic framework, and to disentangle the impact of risk aversion and aversion to fluctuations on the arbitrage between the two types of insurance.

\section{References}

[1] Acemoglu, D. (2009). Introduction to Modern Economic Growth. Princeton University Press.

[2] Arrhenius, O. (1921). Species and Area. Journal of Ecology 9:95-99.

[3] Baumgärtner, S. (2007). The insurance value of biodiversity in the provision of ecosystem services. Natural Resource Modeling 20(1), 87-127.

[4] Baumgärtner, S. and Quaas, M. F. (2010). Managing increasing environmental risks through agrobiodiversity and agrienvironmental policies. Agricultural Economics 41(5), 483-496.

[5] Baumgärtner, S. and Strunz, S. (2014). The economic insurance value of ecosystem resilience. Ecological Economics 101, 21-32.

[6] de Mazancourt, C. Isbell, F., Larocque, A., Berendse, F., De Luca, E., Grace, J. B., Haegeman, B., Wayne P. H., Roscher, C., Schmid, B., Tilman, D., van Ruijven, J., Weigelt, A., Wilsey, B. J. and Loreau, M. (2013). Predicting ecosystem stability from community composition and biodiversity, Ecology Letters 16(5), 617-625. 
[7] Duffie, D. and Epstein, L. G. (1992). Stochastic differential utility. Econometrica, 60(2), 353-94.

[8] Epaulard, A. and Pommeret, A. (2003). Optimally eating a stochastic cake: a recursive utility approach. Resource and Energy Economics 25(2), 129-139.

[9] Epstein, L. and Zin, S. (1989). Substitution, risk aversion and the temporal behavior of consumption and asset returns: a theoretical framework. Econometrica 57(4), 937-969.

[10] Epstein, L. and Zin, S. (1991). Substitution, risk aversion, and the temporal behavior of consumption and asset returns: an empirical analysis. Journal of Political Economy 99(2), 261-286.

[11] Eppink, F. V., and Withagen, C. A. (2009). Spatial patterns of biodiversity conservation in a multiregional general equilibrium model. Resource and Energy Economics, 31(2), 75-88.

[12] Fischer, J., Abson, D. J., Butsic, V., Chappell, M. J., Ekroos, J., Hanspach, J., Kuemmerle, T., Smith, H.G., and von Wehrden, H. (2014). Land sparing versus land sharing: moving forward. Conservation Letters, 7(3), 149-157.

[13] Fuglie, K.O. and Nin-Pratt, A. (2013). Agricultural productivity: a changing global harvest. In "Global food policy report 2013", pages 15-28. International Food Policy Research Institute.

[14] Gleason, H. A. (1922), On the relation between species and area. Ecology 3, 158-162.

[15] Gollier, C. (2001). The Economics of Risk and Time. MIT Press, Cambridge.

[16] Green, R.E., Cornell, S.J., Scharlemann, J.P.W. and Balmford, A. (2005). Farming and the fate of wild nature, Science $307,550-555$.

[17] Ha-Duong, M. and Treich, N. (2004). Risk aversion, intergenerational equity and climate change. Environmental and Resource Economics 28(2), 195-207.

[18] Howitt, R. E., Msangi, S., Reynaud, A. and Knapp, K. C. (2005). Estimating intertemporal preferences for natural resource allocation. American Journal of Agricultural Economics 87(4), 969-983.

[19] Hulme, M. F., Vickery, J. A., Green, R. E., Phalan, B., Chamberlain, D. E., Pomeroy, D. E., Nalwanga, D., Mushabe, D., Katebaka, R., Bolwig, S. and Atkinson, P. W. (2013). Conserving the birds of Uganda's banana-coffee arc: land sparing and land sharing compared. PloS one, 8(2), e54597.

[20] Knapp, K. and Olson, L. (1996). Dynamic resource management: Intertemporal substitution and risk aversion, American Journal of Agricultural Economics 78(4), 1004-1014. 
[21] Krautkraemer, J.A., van Kooten, G.C. and Young, D.L. (1992). Incorporating risk aversion into dynamic programming models, American Journal of Agricultural Economics 74(6), 870-78.

[22] Kremen, C. (2015). Reframing the land-sparing/land-sharing debate for biodiversity conservation. Annals of the New York Academy of Sciences, 1355(1), 52-76.

[23] Lafuite, A.-S. and Loreau, M. (2017). Time-delayed biodiversity feedbacks and the sustainability of social-ecological systems. Ecological Modelling 351, 96-108.

[24] Li, C. Z., Löfgren, K. G., and Weitzman, M. L. (2001). Harvesting versus biodiversity: an Occam's razor version. Environmental and Resource Economics, 18(4), 355-366.

[25] Loreau, M., Mouquet, N. and Gonzalez, A. (2003). Biodiversity as Spatial Insurance in Heterogeneous Landscapes. Proceedings of the National Academy of Sciences of the United States of America 100(22), 12765-12770.

[26] Lucas, R. (1987). Models of business cycles, Blackwell, Oxford.

[27] Lucas, R. (2003). Macroeconomic priorities, American Economic Review 93(1), 1-14.

[28] Lybbert, T.J. and McPeak, J. (2012). Risk and intertemporal substitution: Livestock portfolios and off-take among Kenyan pastoralists. Journal of Development Economics 97(2), 415-426.

[29] Merton, R. C. (1992). Continuous-Time Finance. Wiley-Blackwell.

[30] Peltola, J. and Knapp, K.C. (2001). Recursive preferences in forest management. Forest Science $47(4), 455-465$.

[31] Phalan, B., Bertzky, M., Butchart, S.H.M., Donald, P.F., Scharlemann, J.P.W., Stattersfield, A.J. and Balmford, A. (2013). Crop expansion and conservation priorities in tropical countries. PLoS ONE 8(1):e51759.

[32] Preston, F.W. (1962). The canonical distribution of commonness and rarity: Part I. Ecology $43(2), 185-215$.

[33] Quaas, M.F., Baumgärtner, S., Becker, C., Frank, K. and Mller, B. (2007). Uncertainty and sustainability in the management of rangelands. Ecological Economics 62(2), 251-266.

[34] Quaas, M. F., and Baumgärtner, S. (2008). Natural vs. financial insurance in the management of public-good ecosystems. Ecological Economics 65(2), 397-406.

[35] Renwick, A., and Schellhorn, N. (2016). A perspective on land sparing versus land sharing. in Learning from agri-environment schemes in Australia, 117.

[36] Rosenzweig, M. L. (1995). Species Diversity in Space and Time. Cambridge University Press. 
[37] Smith, W. T. (1996). Feasibility and transversality conditions for models of portfolio choice with non-expected utility in continuous time. Economics Letters 53, 123-131.

[38] Smulders, S., Van Soest, D., and Withagen, C. (2004). International trade, species diversity, and habitat conservation. Journal of Environmental Economics and Management, 48(2), 891-910.

[39] Svennson, L. (1989). Portfolio choice with non-expected utility in continuous-time, Economics Letters $30,313-317$.

[40] Weitzman, M. L. (2000). Economic profitability versus ecological entropy. Quarterly Journal of Economics 115(1), 237-263.

\section{Appendix: Proof of the results}

Proof of Proposition 3.2. Following the approach of [39] (see Proposition 9 and Appendix C of [7] for a more rigorous and detailed mathematical reference), the value function $V$ of our problem evaluated on some trajectory $A(\cdot)$ can be characterized as the solution of the following HamiltonJacobi-Bellman (HJB) equation:

$$
(1-\theta) V(A(t))=\max _{f(t)}\left[(f(t) A(t))^{1-\phi} d t+e^{-\rho d t}\left((1-\theta) \mathbb{E}_{t} V(A(t+d t))\right)^{\frac{1-\phi}{1-\theta}}\right]^{\frac{1-\theta}{1-\phi}} .
$$

By Itô's lemma, we have:

$$
\mathbb{E}_{t} d V(A(t))=\left(V^{\prime}(A(t)) A(t) \alpha+\frac{1}{2} V^{\prime \prime}(A(t)) A(t)^{2} \sigma^{2} f(t)^{\chi}\right) d t=X(A(t), f(t)) d t,
$$

which yields:

$$
(1-\theta) \mathbb{E}_{t} V(A(t+d t))=(1-\theta) V(A(t))\left[1+\frac{X(A(t), f(t))}{V(A(t))} d t\right] .
$$

Using the approximations: $e^{-\rho d t}=1-\rho d t$ and $(1+x d t)^{a}=1+a x d t$, neglecting the terms of order greater than 1 and dropping the time index, the HJB equation may therefore be written as:

$$
\rho \frac{1-\theta}{1-\phi} V(A)=\max _{f}\left[\frac{(f A)^{1-\phi}}{1-\phi} \frac{1}{((1-\theta) V(A))^{\frac{1-\phi}{1-\theta}-1}}+X(A, f)\right] .
$$

Our aim is to prove that the function defined in (11) is a solution of such an equation. We try to find a solution of the form

$$
V(A)=\frac{1}{1-\theta} \beta A^{1-\theta}
$$

for some $\beta>0$. We have $V^{\prime}(A)=\beta A^{-\theta}$ and $V^{\prime \prime}(A)=-\theta \beta A^{-\theta-1}$ so, following $(31), X(A, f)$ specifies as

$$
X(A, f)=\beta A^{1-\theta}\left[\alpha-\theta \frac{\sigma^{2}}{2} f^{\chi}\right]
$$


and $V$ of the prescribed form is a solution of the HJB equation if and only if

$$
\rho \frac{1}{1-\phi} \beta A^{1-\theta}=\max _{f}\left[\frac{(f A)^{1-\phi}}{1-\phi} \frac{1}{\left(\beta A^{1-\theta}\right)^{\frac{1-\phi}{1-\theta}-1}}+\beta A^{1-\theta}\left[\alpha-\theta \frac{\sigma^{2}}{2} f(t)^{\chi}\right]\right]
$$

i.e.

$$
\rho=\max _{f}\left[\frac{f^{1-\phi}}{\beta^{\frac{1-\phi}{1-\theta}}}+(1-\phi)\left[\alpha-\theta \frac{\sigma^{2}}{2} f^{\chi}\right]\right]
$$

i.e.

$$
\rho-\alpha(1-\phi)=\max _{f}\left[\frac{f^{1-\phi}}{\beta^{\frac{1-\phi}{1-\theta}}}-(1-\phi) \theta \frac{\sigma^{2}}{2} f^{\chi}\right] .
$$

The arguments of the maximum in previous expression in $(0,+\infty)$ (which exists since we supposed $\chi>(1-\phi))$ is given by

$$
f^{*}=\left(\frac{1}{\theta \frac{\sigma^{2}}{2} \chi \beta^{\frac{1-\phi}{1-\theta}}}\right)^{\frac{1}{\chi-1+\phi}},
$$

and after finding the expression of $\beta$ we will be able to show that this expression is indeed always in $(0,1)$. If we replace this expression in $(34)$, after some algebra we get the value of $\beta$ that we need to have a solution of the form (33):

$$
\beta=\left[\left(\frac{\chi-1+\phi}{\rho-\alpha(1-\phi)}\right)^{\frac{\chi-1+\phi}{\chi}} \frac{1}{\chi\left(\theta \frac{\sigma^{2}}{2}\right)^{\frac{1-\phi}{\chi}}}\right]^{\frac{1-\theta}{1-\phi}} .
$$

If we use this expression in (35) we obtain

$$
f^{*}=\left[\frac{\rho-\alpha(1-\phi)}{\theta \frac{\sigma^{2}}{2}(\chi-1+\phi)}\right]^{\frac{1}{\chi}} .
$$

Conditions (i) and (ii) of Hypothesis 3.1 and (10) ensure that

$$
0<\frac{\rho-\alpha(1-\phi)}{\theta \frac{\sigma^{2}}{2}(\chi-1+\phi)}<1
$$

so that $f^{*} \in(0,1)$ and all our previous computations are justified ${ }^{8}$.

We finally check the respect of the transversality condition which reads (see [37]):

$$
\lim _{t \rightarrow \infty} \mathbb{E}_{0}\left[e^{-\rho t} V(A(t))\right]=0
$$

Since we have

$$
\mathbb{E}_{0}\left[e^{-\rho t} V(A(t))\right]=\frac{\beta}{1-\theta} \lim _{t \rightarrow \infty} e^{-\rho t} \mathbb{E}_{0}\left[A(t)^{1-\theta}\right]=\frac{\beta}{1-\theta} A_{0} \lim _{t \rightarrow \infty} e^{-\rho t} e^{(1-\theta)\left(\alpha-\theta \frac{\sigma^{2}}{2} f^{\chi}\right) t},
$$

\footnotetext{
${ }^{8}$ Indeed the first part of this inequality is the feasibility condition which (see [37]) is equivalent to the transversality condition for $\theta=\phi$. The second part of the inequality ensures the existence of an interior solution.
} 
the condition (39) is verified whenever $-\rho+(1-\theta)\left(\alpha-\theta \frac{\sigma^{2}}{2} f^{\chi}\right)<0$ i.e., replacing $f^{\chi}$ by its expression given in (37), whenever

$$
\rho\left(1+\frac{\phi-\theta}{\chi}\right)>\alpha(1-\theta)
$$

that is exactly condition (iii) in (9).

Proof of Proposition 3.5. The proof follows the lines of that of Proposition 3.2. Again we have to find a solution of the HJB equation so we need to prove that, if condition (16) is verified, the following equation is satisfied:

$$
\rho \frac{1-\theta}{1-\phi} V(A)=\max _{f \in[0,1]}\left[\frac{(f A)^{1-\phi}}{1-\phi} \frac{1}{((1-\theta) V(A))^{\frac{1-\phi}{1-\theta}-1}}+\left(V^{\prime}(A) A \alpha+\frac{1}{2} V^{\prime \prime}(A) A^{2} \sigma^{2}\right)\right] .
$$

By direct computation we can show that the function

$$
V_{C}(A)=\beta_{C} \frac{A^{1-\theta}}{1-\theta}
$$

with

$$
\beta_{C}=\left[\frac{1}{\rho-(1-\phi)\left(\alpha-\theta \frac{\sigma^{2}}{2}\right)}\right]^{\frac{1-\theta}{1-\phi}}
$$

is a solution. Observe that the argument of the maximum

$$
\max _{f \in[0,1]}\left[\frac{(f A)^{1-\phi}}{1-\phi} \frac{1}{((1-\theta) V(A))^{\frac{1-\phi}{1-\theta}-1}}+\beta_{C} A^{1-\theta}\left(\alpha-\theta \frac{\sigma^{2}}{2} f^{\chi}\right)\right]
$$

is attained (as in the proof of Proposition 3.2) at the the point $\left(\frac{1}{\theta \frac{\sigma^{2}}{2} \chi \beta^{\frac{1-\phi}{1-\theta}}}\right)^{\frac{1}{\chi-1+\phi}}$ when $\theta \frac{\sigma^{2}}{2} \chi \beta_{C}^{\frac{1-\phi}{1-\theta}}>1$ but it is attained at point $f^{*}=1$ when $\theta \frac{\sigma^{2}}{2} \chi \beta_{C}^{\frac{1-\phi}{1-\theta}} \leq 1$. The latter is the case here since, thanks to (16), we have $\rho-\alpha(1-\phi)>\theta \frac{\sigma^{2}}{2}(\chi-1+\phi)$ and then

$$
\theta \frac{\sigma^{2}}{2} \chi \beta_{C}^{\frac{1-\phi}{1-\theta}}=\theta \frac{\sigma^{2}}{2} \chi \frac{1}{\rho-\alpha(1-\phi)+(1-\phi) \theta \frac{\sigma^{2}}{2}} \leq \theta \frac{\sigma^{2}}{2} \chi \frac{1}{\theta \frac{\sigma^{2}}{2}(\chi-1+\phi)+(1-\phi) \theta \frac{\sigma^{2}}{2}}=1 .
$$

So the candidate solution is indeed a solution of the HJB equation if and only if (evaluating the HJB equation at $f=1$ )

$$
\rho \frac{1-\theta}{1-\phi} \beta_{C} \frac{A^{1-\theta}}{1-\theta}=\left[\frac{(A)^{1-\phi}}{1-\phi} \frac{1}{((1-\theta) V(A))^{\frac{1-\phi}{1-\theta}-1}}+\beta_{C} A^{1-\theta}\left[\alpha-\theta \frac{\sigma^{2}}{2}\right]\right] .
$$

Some straightforward calculations allow to prove this relation.

Once we have that we can check the transversality condition as in the proof Proposition 3.2 and then conclude the proof. 
Proof of Proposition 4.2. We can find the result as a corollary of the proofs of Propositions 3.2 and 3.5. Indeed one can think at the welfare in the no-biodiversity case as the optimal welfare in the optimal control problem where the agent can only choose $f=1$. So the candidate-welfare is the solution of the HJB equation (32) evaluated at point $f=1$ i.e.

$$
\rho \frac{1-\theta}{1-\phi} V(A)=\left[\frac{(A)^{1-\phi}}{1-\phi} \frac{1}{((1-\theta) V(A))^{\frac{1-\phi}{1-\theta}-1}}+X(A, 1)\right]
$$

where, as in (31),

$$
X(A(t), 1)=V^{\prime}(A) A \alpha+\frac{1}{2} V^{\prime \prime}(A) A^{2} \sigma^{2} .
$$

By direct computation we can show that the function $V_{B}(A)=\frac{1}{1-\theta} \beta_{B} A^{1-\theta}$ where

$$
\beta_{B}:=\left[\rho-(1-\phi)\left(\alpha-\theta \frac{\sigma^{2}}{2}\right)\right]^{-\frac{1-\theta}{1-\phi}} .
$$

is a solution of this equation. We can then conclude, checking the transversality condition as in the proof Proposition 3.2 and then conclude the proof.

Proof of Proposition 4.3. We use the expressions of $V$ and $V_{C}$ (equations (11) and (18)) to compute $V(A)=V_{C}((1+\lambda) A)$. We obtain:

$$
(1+\lambda)^{1-\theta}=\frac{\beta}{\beta_{C}}
$$

i.e.

$$
\begin{aligned}
(1+\lambda)^{1-\phi} & =\left[\left(\frac{\chi-1+\phi}{\rho-\alpha(1-\phi)}\right)^{\frac{\chi-1+\phi}{\chi}} \frac{1}{\chi\left(\theta \frac{\sigma^{2}}{2}\right)^{\frac{1-\phi}{\chi}}}\right]\left[\rho-(1-\phi)\left(\alpha-\theta \frac{\sigma^{2}}{2}\right)\right] \\
& =\left(\frac{\chi-1+\phi}{\rho-\alpha(1-\phi)}\right)^{\frac{\chi-1+\phi}{\chi}} \frac{1}{\chi\left(\frac{\rho-\alpha(1-\phi)}{(\chi-1+\phi) f^{* \chi}}\right)^{\frac{1-\phi}{\chi}}}\left[\rho-(1-\phi)\left(\alpha-\frac{\rho-\alpha(1-\phi)}{(\chi-1+\phi) f^{* \chi}}\right)\right] \\
& =\frac{\chi-1+\phi}{\chi} f^{*(1-\phi)} \frac{1}{1+\frac{1-\phi}{(\chi-1+\phi) f^{* \chi}}} \\
& =\frac{(\chi-1+\phi) f^{* \chi}+1-\phi}{\chi f^{*}(\chi-1+\phi)}
\end{aligned}
$$

i.e. $(25)$.

Proof of Proposition 4.4. Let

$$
\Lambda=(1+\lambda)^{1-\phi}=\frac{1}{\chi}\left((\chi-1+\phi) f^{*(1-\phi)}+(1-\phi) f^{*(-\chi+1-\phi)}\right) .
$$


We have

$$
\frac{d \Lambda}{d \theta}=\frac{(\chi-1+\phi)(1-\phi)}{\chi} f^{*(-\phi)}\left(1-f^{*(-\chi)}\right) \frac{d f^{*}}{d \theta} .
$$

Besides:

$$
\frac{d \lambda}{d \theta}=\frac{1}{1-\phi} \Lambda^{\frac{1}{1-\phi}-1} \frac{d \Lambda}{d \theta}=\frac{1}{1-\phi}(1+\lambda)^{\phi} \frac{d \Lambda}{d \theta}
$$

Therefore:

$$
\frac{d \lambda}{d \theta}=\frac{\chi-1+\phi}{\chi}\left(\frac{1+\lambda}{f^{*}}\right)^{\phi}\left(1-f^{*(-\chi)}\right) \frac{d f^{*}}{d \theta}
$$

which shows that $\frac{d \lambda}{d \theta}$ is of the opposite sign than $\frac{d f^{*}}{d \theta}$, that is positive. The computation of $\frac{d \lambda}{d \sigma}$ follows exactly the same lines, and the result is the same.

Coming back to $\Lambda$, we can write:

$$
\ln \Lambda=-\ln \chi+(1-\phi) \ln f^{*}+\ln \left(\chi-1+\phi+(1-\phi) f^{*(-\chi)}\right)
$$

which yields:

$$
\frac{1}{\Lambda} \frac{d \Lambda}{d \phi}=-\ln f^{*}+\frac{f^{* \chi}-1}{(\chi-1+\phi) f^{* \chi}+(1-\phi)}+\frac{(\chi-1+\phi)(1-\phi)}{f^{*}} \frac{f^{* \chi}-1}{(\chi-1+\phi) f^{* \chi}+(1-\phi)} \frac{d f^{*}}{d \phi} .
$$

Besides,

$$
\frac{d \lambda}{d \phi}=\frac{1+\lambda}{1-\phi}\left[\frac{1}{\Lambda} \frac{d \Lambda}{d \phi}+\ln (1+\lambda)\right]
$$

which yields:

$$
\frac{d \lambda}{d \phi}=\frac{1+\lambda}{1-\phi}\left[\ln (1+\lambda)-\ln f^{*}+\frac{f^{* \chi}-1}{(\chi-1+\phi) f^{* \chi}+(1-\phi)}+\frac{(\chi-1+\phi)(1-\phi)\left(f^{* \chi}-1\right)}{f^{*}\left[(\chi-1+\phi) f^{* \chi}+(1-\phi)\right]} \frac{d f^{*}}{d \phi}\right]
$$

which sign is at first glance ambiguous, and proves to be very difficult to determine analytically.

Proof of Lemma 4.5. It is sufficient to use the Taylor expansion of the expression of $\lambda$. The first order term vanishes while the second gives the claim.

Proof of Proposition 4.7. As a first step we calculate the expression of $\Pi(A, f)$ (recall that $f$ is fixed). Given the linearity of consumption in terms of productivity and of productivity in terms of the initial datum, we will look in particular for a linear $\Pi$ as a function of $A$. The equation which needs to be satisfied is

$$
\mathcal{W}\left(f \mathbb{E}\left(\left(A_{f}(\cdot)-\Pi\left(A_{f}(\cdot), f\right)\right)\right)=\mathcal{W}\left(f A_{f}(\cdot)\right) .\right.
$$

The right hand side of this equation is the welfare obtained starting from $A$ always using a fraction $f$ of the land for agriculture. Consistently with previous notations we call it $V_{f}(A)$. As in the proof of Proposition 4.2 we observe that its expression can be found as a corollary of the proofs of Propositions 3.2 and 3.5 since the welfare obtained for a fixed pre-specified $f$ can be seen as the optimal welfare 
in the optimal control problem where the agent can only choose this $f$. Thus, as a first step, we look for a solution of the HJB equation (32) evaluated at point $f$ :

$$
\rho \frac{1-\theta}{1-\phi} V(A)=\frac{(f A)^{1-\phi}}{1-\phi} \frac{1}{((1-\theta) V(A))^{\frac{1-\phi}{1-\theta}-1}}+\left(V^{\prime}(A) A \alpha+\frac{1}{2} V^{\prime \prime}(A) A^{2} \sigma^{2} f^{\chi}\right) .
$$

Arguing as in the proofs of Propositions 3.2, 3.5 and 4.2 we can directly check that the function

$$
V_{f}(A)=\beta_{f} \frac{A^{1-\theta}}{1-\theta}
$$

where

$$
\beta_{f}=\frac{f^{1-\theta}}{\left[\rho-\alpha(1-\phi)+(1-\phi) \theta \frac{\sigma^{2}}{2} f^{\chi}\right]^{\frac{1-\theta}{1-\phi}}},
$$

is a solution of (5). We can check the transversality condition as in the proof Proposition 3.2 so we finally have that $V_{f}$ is the welfare we were looking for.

We need now to compute the left hand side of (43). Let $Z(t)=\mathbb{E}\left(\left(A_{f}(t)-\Pi\left(A_{f}(t), f\right)\right) . Z(t)\right.$ is certain and, since we are looking for a $\Pi$ which is a linear function of $A, d Z(t)=\alpha Z(t) d t$. Since the consumption $C(t)$ is equal to $f Z(t)$ the welfare process can be characterized as the solution of the following HJB equation:

$$
\left.((1-\theta) V(Z(t)))^{\frac{1-\phi}{1-\theta}}=(f Z(t))\right)^{1-\phi} d t+e^{-\rho d t}\left[(1-\theta) \mathbb{E}_{t} V(Z(t+d t))\right]^{\frac{1-\phi}{1-\theta}} .
$$

Using that

$$
\mathbb{E}_{t} V(Z(t+d t))=V(Z(t))+\mathbb{E}_{t} d V(Z(t))=V(Z(t))+V^{\prime}(Z(t)) Z(t) \alpha d t
$$

and dropping the time index we get

$$
\begin{aligned}
& ((1-\theta) V(Z))^{\frac{1-\phi}{1-\theta}} \\
& =(f Z)^{1-\phi} d t+e^{-\rho d t}\left[(1-\theta) V(Z)+V^{\prime}(Z) Z \alpha d t\right]^{\frac{1-\phi}{1-\theta}} \\
& =(f Z)^{1-\phi} d t+(1-\rho d t)((1-\theta) V(Z))^{\frac{1-\phi}{1-\theta}}\left[1+\frac{V^{\prime}(Z) Z \alpha}{V(Z)} d t\right]^{\frac{1-\phi}{1-\theta}} \\
& =(f Z)^{1-\phi} d t+(1-\rho d t)((1-\theta) V(Z))^{\frac{1-\phi}{1-\theta}}+\frac{1-\phi}{1-\theta}((1-\theta) V(Z))^{\frac{1-\phi}{1-\theta}} \frac{V^{\prime}(Z) Z \alpha}{V(Z)} d t
\end{aligned}
$$

i.e.

$$
0=(f Z)^{1-\phi}-\rho((1-\theta) V(Z))^{\frac{1-\phi}{1-\theta}}+\frac{1-\phi}{1-\theta}((1-\theta) V(Z))^{\frac{1-\phi}{1-\theta}} \frac{V^{\prime}(Z) Z \alpha}{V(Z)} .
$$

We look for a $1-\theta$-homogeneous solution of the form $V_{e}(A)=\beta_{e} \frac{A^{1-\theta}}{1-\theta}$. The HJB equation above can have a solution of this form if and only if

$$
\begin{aligned}
0 & =(f Z)^{1-\phi}-\rho\left(\beta_{e} Z^{1-\theta}\right)^{\frac{1-\phi}{1-\theta}}+(1-\phi)\left(\beta_{e} Z^{1-\theta}\right)^{\frac{1-\phi}{1-\theta}} \alpha \\
& =f^{1-\phi} Z^{1-\phi}-\beta_{e}^{\frac{1-\phi}{1-\theta}} Z^{1-\phi}(\rho-\alpha(1-\phi))
\end{aligned}
$$


i.e.

$$
f^{1-\phi}=\beta^{\frac{1-\phi}{1-\theta}}(\rho-\alpha(1-\phi))
$$

which yields:

$$
\beta_{e}=f^{1-\theta}\left[\frac{1}{\rho-\alpha(1-\phi)}\right]^{\frac{1-\theta}{1-\phi}}
$$

and

$$
V_{e}(Z)=\frac{(f Z)^{1-\theta}}{1-\theta}\left[\frac{1}{\rho-\alpha(1-\phi)}\right]^{\frac{1-\theta}{1-\phi}}
$$

which is the expression of the left hand side of (43). Finally, using equations (45) and (44), we can see that (43) is verified if and only if

$$
\frac{(f(A-\Pi))^{1-\theta}}{1-\theta}\left[\frac{1}{\rho-\alpha(1-\phi)}\right]^{\frac{1-\theta}{1-\phi}}=\frac{(f A)^{1-\theta}}{(1-\theta)\left[\rho-(1-\phi)\left(\alpha-\theta \frac{\sigma^{2}}{2} f^{\chi}\right)\right]^{\frac{1-\theta}{1-\phi}}}
$$

which gives, after some computations,

$$
\Pi(A, f)=A\left[1-\left(\frac{\rho-\alpha(1-\phi)}{\rho-(1-\phi)\left(\alpha-\theta \frac{\sigma^{2}}{2} f \chi\right)}\right)^{\frac{1}{1-\phi}}\right] .
$$

This gives the expression of $\Pi$ (linear in $A$ and depending on $f$ and all various parameters) we were looking for. We can now use it to find the explicit expression of the insurance value of biodiversity. First we compute its derivative with respect to $f$ :

$$
\frac{\partial \Pi(A, f)}{\partial f}=A \frac{1}{1-\phi}(\rho-\alpha(1-\phi))^{\frac{1}{1-\phi}}\left(\rho-(1-\phi)\left(\alpha-\theta \frac{\sigma^{2}}{2} f^{\chi}\right)\right)^{-\frac{1}{1-\phi}-1}(1-\phi) \theta \frac{\sigma^{2}}{2} \chi f^{\chi-1} .
$$

and then we compute such a derivative at point $f=f^{*}$ (found in Proposition 3.2). After some algebra we obtain

$$
v(A)=-\left.\frac{\partial \Pi(A, f)}{\partial(1-f)}\right|_{f=f^{*}}=\left.\frac{\partial \Pi(A, f)}{\partial f}\right|_{f=f^{*}}=A\left(\frac{\chi-1+\phi}{\chi}\right)^{\frac{1}{1-\phi}}\left(\frac{\theta \frac{\sigma^{2}}{2}(\chi-1+\phi)}{\rho-\alpha(1-\phi)}\right)^{\frac{1}{\chi}} .
$$

Proof of Proposition 4.8. We easily see that:

$$
\frac{d v(A) / A}{d \theta}=-\left(\frac{\chi-1+\phi}{\chi}\right)^{\frac{1}{1-\phi}} \frac{1}{f^{*}} \frac{d f^{*}}{d \theta},
$$

which shows that $\frac{d v(A) / A}{d \theta}$ is of the opposite sign than $\frac{d f^{*}}{d \theta}$, that is positive. The computation of $\frac{d v(A) / A}{d \sigma}$ follows exactly the same lines, and the result is the same.

We have: $\ln \frac{v(A)}{A}=\frac{1}{1-\phi} \ln \frac{\chi-1+\phi}{\chi}-\ln f^{*}$, which yields:

$$
\frac{1}{v(A) / A} \frac{d v(A) / A}{d \phi}=\frac{1}{(1-\phi)^{2}} \ln \frac{\chi-1+\phi}{\chi}+\frac{1}{(1-\phi)(\chi-1+\phi)}-\frac{1}{f^{*}} \frac{d f^{*}}{d \phi} .
$$


A study of the function of $\phi$ and $\chi$ formed by the first two terms of this equation shows that it is always positive. Therefore, a sufficient conditions for which $\frac{d v(A) / A}{d \phi}$ to be positive is $\alpha \chi-\rho<0$, which ensures that $\frac{d f^{*}}{d \phi}<0$. If this condition is not satisfied, the sign of $\frac{d v(A) / A}{d \phi}$ is ambiguous. 